\title{
A Vapor Sensor Based on a Porous Silicon Microcavity for the Determination of Solvent Solutions
}

\author{
Huy Bui ${ }^{1 *}$, Thuy Van Nguyen ${ }^{1}$, The Anh Nguyen ${ }^{1}$, Thanh Binh Pham ${ }^{1}$, Quoc Trung Dang ${ }^{1}$, \\ Thuy Chi Do ${ }^{2}$, Quang Minh Ngo ${ }^{1}$, Roberto Coisson ${ }^{3}$, and Van Hoi Pham ${ }^{1}$ \\ ${ }^{1}$ Institute of Materials Science, VAST, 18 Hoang Quoc Viet Rd., Cau Giay Dist., 10000 Hanoi, Vietnam \\ ${ }^{2}$ Thai Nguyen University of Education, 20 Luong Ngoc Quyen road, 23000 Thai Nguyen, Vietnam \\ ${ }^{3}$ Department of Physics and Earth Science, Parma University, Viale delle Scienze, 43100 Parma, Italy
}

(Received May 13, 2014 : revised July 4, 2014 : accepted July 25, 2014)

\begin{abstract}
A porous silicon microcavity (PSMC) sensor has been made for vapors of solvent solutions, and a method has been developed in order to obtain simultaneous determination of two volatile substances with different concentrations. In our work, the temperature of the solution and the velocity of the air stream flowing through the solution have been used to control the response of the sensor for ethanol and acetone solutions. We study the dependence of the cavity-resonant wavelength shift on solvent concentration, velocity of the airflow and solution temperature. The wavelength shift depends linearly on concentration and increases with solution temperature and velocity of the airflow. The dependence of the wavelength shift on the solution temperature in the measurement contains properties of the temperature dependence of the solvent vapor pressure, which characterizes each solvent. As a result, the dependence of the wavelength shift on the solution temperature discriminates between solutions of ethanol and acetone with different concentrations. This suggests a possibility for the simultaneous determination of the volatile substances and their concentrations.
\end{abstract}

Keywords : Porous silicon, Microcavity, Optical sensors, Solvent, Electrochemical etching OCIS codes : (040.6040) Silicon; (140.3945) Microcavities; (130.6010) Sensors

\section{INTRODUCTION}

Owing to high specific surface area [1], porous silicon (PS) is an ideal transducer material for sensors of liquids [2-4] and vapors [5, 6]. Recently, PS optical sensors have been designed in the structure of one-dimensional photonic crystal devices such as optical filters [7] and microcavities [8]. The principle of these sensors is a determination of the photonic crystal spectral shift caused by refractive index change of the nano-porous silicon layers in the device due to the interaction with liquid or gas. It is shown from the principle that the response of the sensor is only dependent on refractive index and therefore lacks specificity for the studied substances. Consequently, most of current sensors based on the PS photonic crystal only determine the concentration of a defined substance. It is possible to use a physical or chemical method to overcome this drawback. A commonly used chemical method is the functionalization of the surface of silicon nanocrystals in the porous layers $[9,10]$. It is a chemical process to create the new chemical bond that combines selectively with molecules of the studied substances. The number of published works, which used a physical method to identify the analyses, in sensors based on photonic crystals has been limited. Sailor applied temperature cycles to a porous silica photonic crystal embedded in pure chemical vapors and therefore he was able to distinguish between isopropanol, heptane, and cyclohexane [11], Ye presented a multi-beam interferometry method using optical fibers for detection of methane gas with concentration sensitivity of $0.015 \%$ [12]. The sensitivity of optical sensors is defined as the ratio of the wavelength shift and the change of the ambience refractive index, and depends on the concentration change of the solution, can be enhanced by designing suitable

\footnotetext{
*Corresponding author: buihuy@ims.vast.ac.vn

Color versions of one or more of the figures in this paper are available online.
} 
structural parameters such as thickness, porosity, number of porous layers in the device [13] or creating a stress on the sensor surface [14].

This paper presents the use of a vapor sensor based on a porous silicon microcavity for the determination of solvent solutions. We set up the measurement, in which the temperature of the solution and the velocity of the airflow containing the solvent gas from solutions of ethanol and acetone control the response of the sensor. As mentioned above, the sensor uses the physico-chemical properties of the analyzed substances as "characteristic signals" involved in the response of the sensor. The sensor response is given by the shift of the resonant wavelength of the microcavity when sensors are immersed in the flow of solvent vapor. We present a study of the dependence of the wavelength shift on solvent concentration, velocity of the airflow and solution temperature. Considering those dependencies, we hope to find out the potential to enhance the sensitivity of the sensors and the specificity of the measurement.

\section{EXPERIMENTAL}

Porous silicon microcavities were fabricated by an electrochemical method in a process that was presented in our previous work [15]. In particular, the electrochemical process was carried out on a (100)-oriented boron-highly doped p-type $\mathrm{Si}$ wafer (resistivity is of $0.01-0.1 \Omega . \mathrm{cm}$ ) in a 16\% hydrofluoric acid (HF) solution and ethanol at various current densities. Aluminum was evaporated onto the backside of the $\mathrm{Si}$ wafer and then it was annealed at $420^{\circ} \mathrm{C}$ in nitrogen atmosphere for $45 \mathrm{~min}$. in order to ensure a good Ohmic contact. The electrochemical process was controlled by computer program using Galvanostat equipment (Autolab PGSTAT 30) so precise control over electrical current and etching time was achieved. Before electrochemical etching, the $\mathrm{Si}$ wafer was dipped in $5 \%$ HF solution for a minute to remove the native oxide. The electrochemical anodization cell was made of polytetrafluoroethylene (Teflon) resin and was designed to have an exposed etching area of approximately $0.79 \mathrm{~cm}^{2}$. After anodization, the sample was washed with $98 \%$ ethanol and dried in primary vacuum. For converting the surface of the silicon nano-crystals from hydrophobic to hydrophilic, we oxidized the as-prepared sample in an ozone atmosphere for $45 \mathrm{~min}$. by using the ozone generator (H01 BKOzone with a capacity of $500 \mathrm{mg}$ /hour).

Cross-sectional and top view images of the porous silicon microcavity were obtained using an ultra resolution field-emission scanning electron microscope (FE-SEM) S-4800. Figure 1 shows plan-view and cross-section images of the microcavity based on $(\mathrm{HL})^{3,5} \mathrm{LL}(\mathrm{HL})^{3}$ porous silicon multilayer structure, where $\mathrm{H}$ and $\mathrm{L}$ labels correspond to high and low refractive index layers, respectively, 3.5 means three and half pairs of HL. We choose structure with 3 and 3.5 pairs of $\mathrm{HL}$, because this gives a good reflectivity spectrum and easily repeatable electrochemical etching process. The thicknesses of high and low refractive layers were $72 \mathrm{~nm}$ and $87 \mathrm{~nm}$ with accuracy of $\pm 2 \mathrm{~nm}$, respectively. This structure was obtained from anodization current density of $15 \mathrm{~mA} / \mathrm{cm}^{2}$ and $50 \mathrm{~mA} / \mathrm{cm}^{2}$ and with etching time $5.56 \mathrm{sec}$ and $2.86 \mathrm{sec}$ for high and low refractive index layers, respectively. For measurement of reflective spectra of the samples, we used a spectrometer (S-2000, Ocean Optics) with a resolution of $0.4 \mathrm{~nm}$ and a tungsten halogen lamp (Z 19, Narva).

Figure 2 shows the reflectivity spectra of the microcavity before and after oxidization. The blue shift of the resonant wavelength after oxidization is due to a decrease in the effect of refractive index of the porous layers in the microcavity [16]. From experimental results we calculated refractive indices of 2.1 and 1.75 for high and low

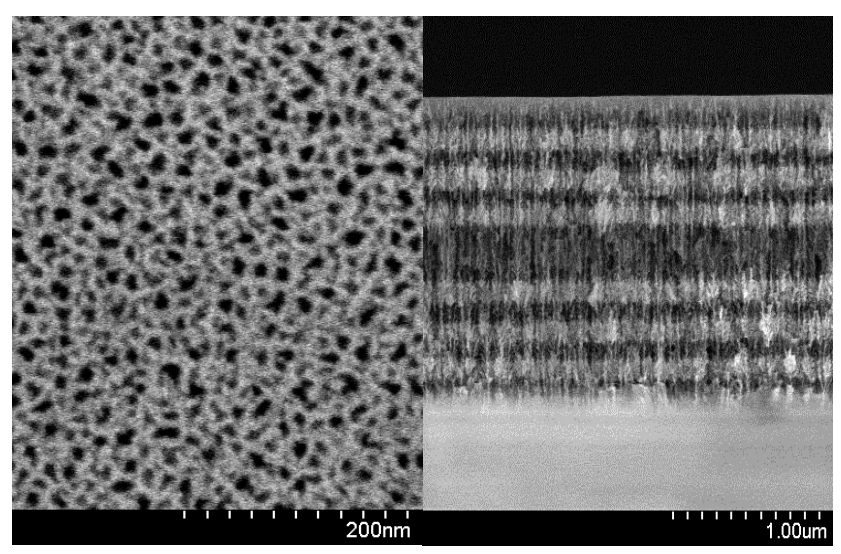

(a)

(b)

FIG. 1. (a) SEM plan-view and (b) cross-section images of a microcavity design in the $(\mathrm{HL})^{3.5} \mathrm{LL}(\mathrm{HL})^{3}$ structure.

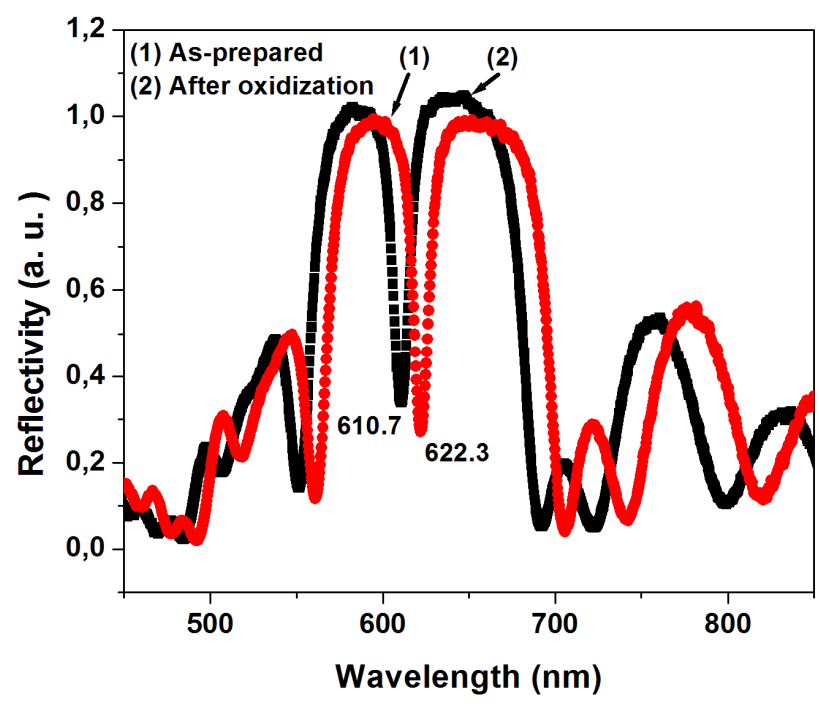

FIG. 2. Reflective spectra of the porous silicon microcavity as-prepared (curve 1) and after oxidization in the ozone ambience (curve 2). 


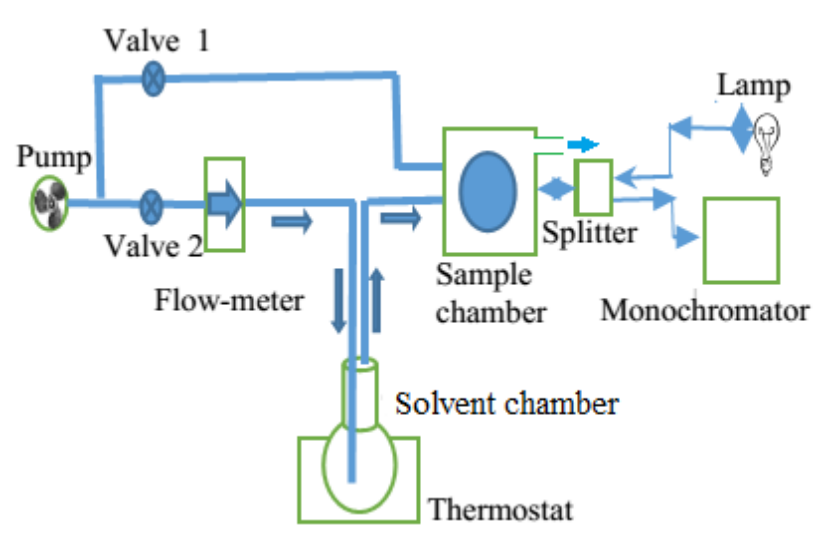

FIG. 3. Schematic of the concentration measurement for volatile organics by using a vapor sensor based on the porous silicon microcavity. Valve 1 and valve 2 are used to guide the airflow from a pump into the sample chamber. The arrows indicate the direction of the air or vapor flows.

refractive index layers, respectively.

Figure 3 shows the schematic of the concentration measurement for volatile organics by using a vapor sensor based on a porous silicon microcavity. In this schematic, valve 2 works as a controller of velocity of air stream though the flow meter, the test solvent chamber and the sample chamber. Valve 1 is only open to refresh the porous matrix after measurement. The thermostat controls the temperature of the liquid in the range from room temperature to $100^{\circ} \mathrm{C}$.

In our experiment, we use an optical fiber splitter BIF200 UV-VIS for light irradiation to samples and for collecting the reflective spectrum of the microcavity. We have also used a LM35D integrated circuit for measuring the temperature in the sample chamber. It is shown that this temperature was not affected by the solution temperature nor by the air rate in our experimental setup. For an experimental data run, it takes $5 \mathrm{~min}$. to $7 \mathrm{~min}$. depending on the velocity of the airflow. A standard deviation of the wavelength shift from the average value of 5 experimental data runs is $0.6 \mathrm{~nm}$.

\section{RESULTS AND DISCUSSION}

It is known that the response of the sensor depends on solvent vapor pressure in the sensor chamber [17]. This vapor pressure is related to the vapor pressure of the solvent in the solution chamber through a gas stream flowed through the solution. Assuming that the vapor pressure in the solution chamber obeys the rules of vapor pressure in a closed system, the relation between the wavelength shift $(\Delta \lambda)$, the vapor pressure in the solution chamber $\left(\mathrm{P}_{\text {solution. }}\right)$ and the velocity of airflow $(\mathrm{V})$ is crudely presented as $\Delta \lambda \sim P_{\text {solution }} \vartheta \vartheta(V)$, where $\vartheta(V)$ is an empirical function of $V$, which shows dependence of concentration of solution on the velocity of airflow. The $\mathrm{P}$ solution can be calculated by following formulas [18]:

$$
\begin{aligned}
& P_{\text {solution }}=\sum_{i}\left(P_{i} \cdot X_{i}\right) \text { (Raoul's law) } \\
& \log _{10} P_{i}=A-\frac{B}{C+T} \text { (Antoine equation) }
\end{aligned}
$$

Where $P_{i}$ is the vapor pressure of a particular substance, $X_{i}$ is the corresponding mole fraction of that substance, $A$,

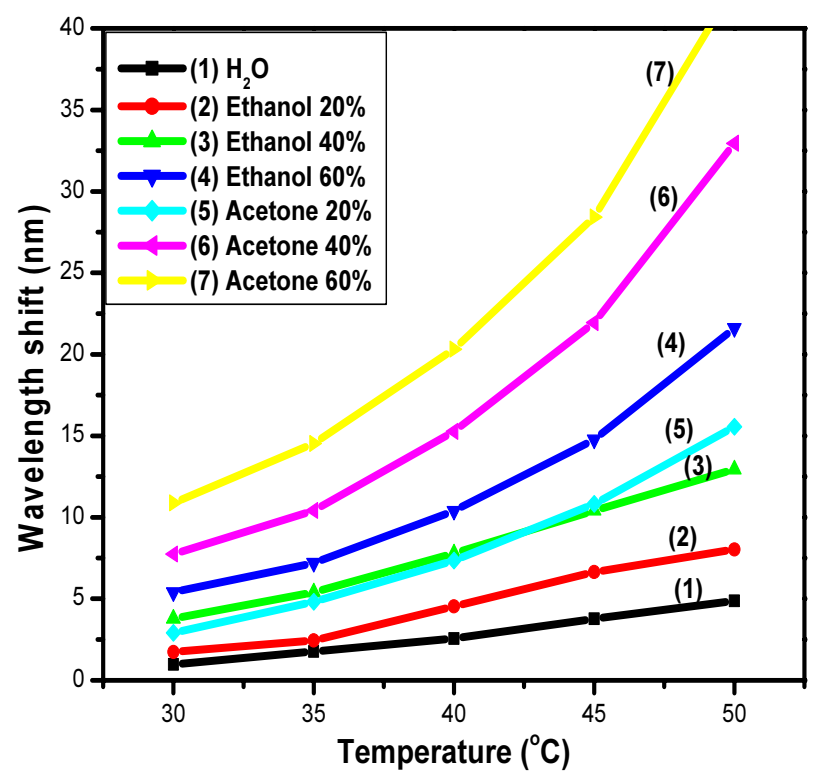

FIG. 4. The dependence of the wavelength shift on the solution temperature for pure $\mathrm{H}_{2} \mathrm{O}$ (curve 1), 20 - 60\% ethanol (curves 2-4), and 20-60\% acetone (curves 5-7) at $\mathrm{V}=$ $0.84 \mathrm{ml} . \mathrm{s}^{-1}$.

TABLE 1. Physical properties of acetone and ethanol

\begin{tabular}{c|c|c|c|c|c|c|c|c}
\hline \hline \multirow{2}{*}{ Solvent } & \multirow{2}{*}{$\begin{array}{c}\text { Boiling point } \\
\left({ }^{\circ} \mathrm{C}\right)\end{array}$} & \multirow{2}{*}{$\begin{array}{c}\text { Refractive } \\
\text { index }\end{array}$} & \multicolumn{2}{|c|}{$\begin{array}{c}\text { The coefficients of Antoine's } \\
\text { equation }\end{array}$} & \multicolumn{2}{|c|}{$\left(^{*}\right) \mathrm{P}_{\mathrm{i}}$} & \multicolumn{2}{|c}{$(*) \mathrm{dP} / \mathrm{dT}\left(\mathrm{mmHg} /{ }^{\circ} \mathrm{C}\right)$} \\
\cline { 4 - 9 } & & $\mathrm{A}$ & $\mathrm{B}$ & $\mathrm{C}$ & $\mathrm{T}=30^{\circ} \mathrm{C}$ & $\mathrm{T}=30^{\circ} \mathrm{C}$ & $\mathrm{T}=50^{\circ} \mathrm{C}$ \\
\hline Ethanol & 78.5 & 1.361 & 8.05 & 1554.3 & 222.65 & 78.154 & 480.802 & 1259.4 \\
\hline Acetone & 56.2 & 1.359 & 7.03 & 1161.0 & 224 & 284.187 & 1299.00 & 2596.1 \\
\hline
\end{tabular}

(*) Calculated results by using Antoine's equation (3) 
$B$ and $C$ are component-specific constants (the coefficients of Antoine's equation), "i" is an indexing component that keeps tract of each substance in the solution.

The equations (1-2) show that $\Delta \lambda$ is a function of $V, X_{\mathrm{i}}$ and $P_{i}(T)$. Below we consider those relations in the experiment.

As mentioned above, we carried out experiments on ethanol and acetone solution. These are very common organic solvents and some of their physical properties such as boiling point, refractive indices and Antoine's coefficients from [18] are shown in Table 1.

Figure 4 shows the dependence of $\Delta \lambda$ on $T, \Delta \lambda(T)$, for acetone and ethanol solutions with various concentrations at the airflow velocity of $0.84 \mathrm{ml} . \mathrm{s}^{-1}$. The equations (1-2) show that we can consider the temperature dependence of $\Delta \lambda(T)$ as the temperature dependence of $P_{i}(T)$ modified by multipliers $X_{\mathrm{i}}$ if it was assumed that the contribution of water to solution pressure is small. Experimental data from curve 1 , which describes $\Delta \lambda(T)$ of the water, shows the validity of this assumption in our measurement. $P_{i}(T)$ steadily increases as temperature increases (i.e. a monotonically increasing function), so the curves of solvent solutions with various concentrations are separate, for example the curves 2-4 of ethanol solutions or the curves 5-7 of acetone solutions. Using equation (2) we calculated the rate of change of $P_{i}(T)$ for acetone and ethanol in the range of temperature from $30^{\circ} \mathrm{C}$ to $50^{\circ} \mathrm{C}$ and its values presented in the Table 1.

The slope of $P_{\text {acetone }}(T)$ is greater than that of $P_{\text {ethanol }}$ (T) in the studied range of temperature (see in the Table $1)$, so the curves describing $\Delta \lambda(T)$ of acetone and ethanol solutions are intersecting with each other not more than one time (for example: the curves 3 and 5), or not intersecting (for example: the curves 3 and 4). Consequently, a curve describing $\Delta \lambda(T)$ characterizes the solution of acetone (or ethanol) at a given concentration. In other words, the dependence of the wavelength shift on the solution temperature discriminates between solutions of ethanol and acetone with various concentrations.

As our previous work [4], we have tested the response of the sensors immersed to liquid phase solution of $100 \%$ acetone and denatured ethanol using a Cary 5000 spectrometer system with a resolution of $0.1 \mathrm{~nm}$. A difference of 0.5 $\mathrm{nm}$ in the resonance wavelength shift of cavity for these two solvents was obtained due to the small difference in their refractive indices.

Figure 5 shows the dependence of the resonant wavelength shift $\Delta \lambda(\mathrm{C})$ on ethanol concentration, when velocity of the airflow $(V)$ and temperature of the solution $(T)$ work as parameters in the measurements. It can be seen in Figure 5 that the curve described by $\Delta \lambda(\mathrm{C})$ is linear and its slope, i.e. sensitivity of the measurement, increases as $V$ and $T$ increase. Those remarks are also deduced from equations (1-2) when $X_{i}$ is a variable, and $T$ and $V$ are parameters. Linearity of this dependence is a favorable condition for determination of solvent concentration. The increase of the slope creates an increase in sensitivity received from the measurement. From data in the curves 2 and 3 , which were received from the measurement with parameters $T$ and $V$ at $45^{\circ} \mathrm{C}$ and $0.84 \mathrm{ml} . \mathrm{s}^{-1}$, and at $30^{\circ} \mathrm{C}$ and $1.68 \mathrm{ml} . \mathrm{s}^{-1}$, we obtain the difference of $\Delta \lambda$ of about $18.5 \mathrm{~nm}$ and $10.0 \mathrm{~nm}$, respectively, between the $0 \%$ and $100 \%$ ethanol. Whereas having measured with this sensor in the liquid phase in this concentration range, we obtained the $\Delta \lambda$ difference of about $5 \mathrm{~nm}$ only [19].

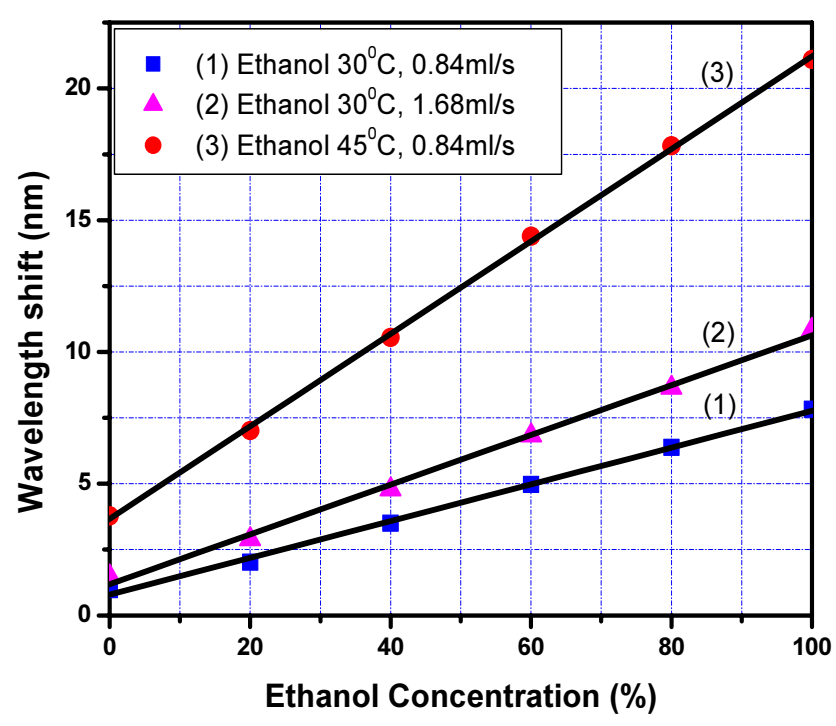

FIG. 5. The dependence of the wavelength shift on ethanol concentration when the velocity of air flow $(V)$ and temperature of solution $(T)$ work as parameters in the measurements. The curves 1-3 received from measurements with pairs of these parameters such as $V=0.84 \mathrm{ml}^{-1} \mathrm{~s}^{-1}$ and $T=30^{\circ} \mathrm{C}, V=0.84 \mathrm{ml} . \mathrm{s}^{-1}, T=45^{\circ} \mathrm{C}, V=1.68 \mathrm{ml} . \mathrm{s}^{-1}$ and $\mathrm{T}=30^{\circ} \mathrm{C}$, respectively.

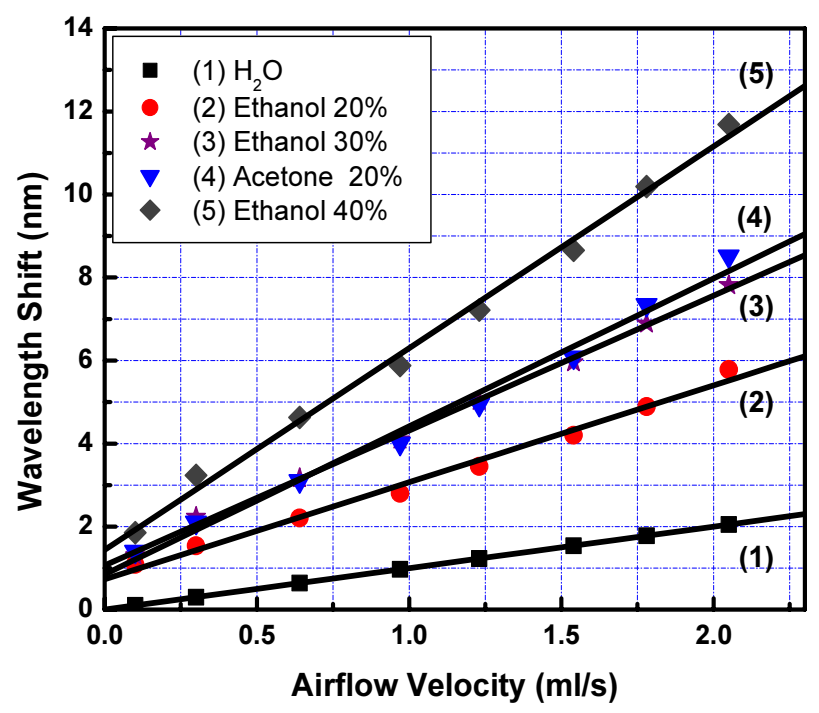

FIG. 6. The dependence of the wavelength shift on the airflow velocity in the range $0-2.5 \mathrm{ml} . \mathrm{s}^{-1}$. 
Therefore, the sensitivity received from the measurement in the vapor phase with the value of $T$ and $V$ at $45^{\circ} \mathrm{C}$ and $0.84 \mathrm{ml} . \mathrm{s}^{-1}$, and at $30^{\circ} \mathrm{C}$ and $1.68 \mathrm{ml} . \mathrm{s}^{-1}$ increases 3.7 and 2.0 times, respectively, as compared with that in the liquid phase. We expect that the sensitivity of the measurement can be strongly improved with a reasonable combination of both parameters $T$ and $V$.

Figure 6 shows the dependence of $\Delta \lambda$ on $\mathrm{V}, \Delta \lambda(\mathrm{V})$, at a temperature of $30^{\circ} \mathrm{C}$ when concentration of ethanol and acetone work as the parameters. It can be seen in Fig. 6 that curves describing $\Delta \lambda(\mathrm{V})$ are separate straight lines with different concentrations of acetone and ethanol. This shows that empirical function $\vartheta(V)$ is a linear function of $\mathrm{V}$. Now, we consider properties of the slopes of curves in Fig. 6. According to equation (1), slope of the curve describing $\Delta \lambda(V)$ increases as $P_{i}$ and $X_{i}$ increase. We apply the obtained results for curves 2 and 3 received from measurements with ethanol and acetone solutions at the same concentration $(20 \%)$. It can be seen that the vapor pressure of acetone is larger than that of ethanol (see in the Table 1), so the slope of curve 3 is larger than that of curve 2 .

We also apply those results for curve 2 and 4 received from measurements with ethanol concentrations at $40 \%$ and $20 \%$. Slope of curve 4 is larger than that of curve 2 due to the greater value in concentration. It is deduced from Fig. 6 that dependence of the wavelength shift on velocity of the airflow is linear, and the slopes $\Delta \lambda \Delta V$ are $2.4 \mathrm{~nm} / \mathrm{ml} . \mathrm{s}^{-1}$ and $3.7 \mathrm{~nm} / \mathrm{ml}^{-\mathrm{s}^{-1}}$ for the same concentration of $20 \%$ ethanol and acetone solutions, respectively. In addition, when the concentration of organic solvent increases, the slopes $\triangle N \Delta V$ would be enhanced (for example, the value of $\Delta \lambda \Delta V$ enhanced from $2.4 \mathrm{~nm} / \mathrm{mll}^{-1}$ to 3.4 and $5.1 \mathrm{~nm} / \mathrm{ml} . \mathrm{s}^{1}$ when the concentration of ethanol increased from $20 \%$ to $30 \%$ and $40 \%$, respectively). Based on this phenomenon, we can simultaneously determine the kind and concentration of organic content in the solutions. For example, $40 \%$ ethanol and $20 \%$ acetone have similar temperature dependence (see Fig. 4) but can be discriminated by their air flow velocity dependence, and while 30\% ethanol and $20 \%$ acetone have similar air flow velocity dependence (as can be seen from Fig. 6), they can be discriminated by their temperature dependence.

\section{CONCLUSION}

In conclusion, we successfully built a high sensitivity measurement system for the determination of solvent solutions by using a vapor sensor based on a porous silicon microcavity. In this measurement, the sensor response is controlled by temperature of the solution and velocity of the air stream flowing though the solution. We studied the dependence of the wavelength shift on solvent concentration, velocity of the airflow and temperature solution for the solutions of ethanol and acetone with various concentrations and in order to enhance the sensitivity and specificity of the measurement. The dependence of the wavelength shift on concentration is linear and sensor sensitivity increases with temperature of the solution and velocity of the air stream. Solution temperature and air flow velocity determine the equilibrium of partial vapor condensation in the pores and then contain characteristics of specific solvent vapor pressure and liquid refractive index that allows discrimination between ethanol and acetone and determine the concentration. This suggests a possibility for simultaneous determination concentration and type of solvent.

\section{ACKNOWLEDGMENT}

This work financially supported by National Foundation for Science and Technology Development (NAFOSTED) of Vietnam under Grant No.103.06.38.09. This work had been using the apparatus of National Key Laboratory for Electronic Materials and Devices in Institute of Materials Science.

\section{REFERENCES}

1. S. C. Bayliss, "The structure of porous silicon from XAFS and ES," in Structural and Optical Properties of Porous Silicon Nanostructures, G. Amato, C. Delerue, and H. J. von Bardeleben, ed. (Gordon and Breach Science Publishers, Indian, 1997), Chapter 12.

2. J. P. Badilla, D. C. Rojas, V. Lopez, B. D. Fahlman, and A. Ramirez-Porras, "Development of an organic vapor sensor based on functionalized porous silicon," Phys. Stat. Sol. (a) 208, 1458-1461 (2011).

3. L. De Stefano, L. Moretti, I. Rendina, and A. M. Rosi, "Porous silicon microcavities for optical hydrocarbons detection," Sens. Actuators A 104, 179-182 (2003).

4. V. H. Pham, H. Bui, L. H. Hoang, T. V. Nguyen, T. A. Nguyen, T. S. Pham, and Q. M. Ngo, "Nano-porous silicon microcavity sensors for determination of organic fuel mixtures," J. Opt. Soc. Korea 17, 423-427 (2013).

5. L. De Stefano, K. Malecki, F. G. Della Corte, L. Moretti, I. Rea, L. Rotiroti, and I. Rendina, "A microsystem based on porous silicon-glass anodic bonding for gas and liquid optical sensing," Sensors 6, 680-687 (2006).

6. H. J. Kim, Y. Y. Kim, K. W. Lee, and S. H. Park, "A distributed Bragg reflector porous silicon layer for optical interferometric sensing of organic vapor," Sens. Actuators B 155, 673-678 (2011).

7. T. Jalkanen, V. Torres-Costa, J. Salonen, M. Bjürkqvist, E. Mökilö, J. M. Martinez-Duart, and V. P. Lehto, "Optical gas sensing properties of thermally hydrocarbonized porous silicon Bragg reflectors," Opt. Express 17, 5446-5456 (2009).

8. L. De Stefano, L. Moretti, I. Rendina, and A. M. Rossi, "Quantitative optical sensing in two-component mixtures using porous silicon microcavities," Phys. Stat. Sol. (a) 201, 1011-1016 (2004).

9. O. Meskini, A. Abdelghani, A. Tlili, R. Mgaieth, N. Jaffrezic- 
Renault, and C. Martelet, "Porous silicon as functionalized material for immunosensor application," Talanta 71, 14301433 (2007).

10. M. Hiraoui, M. Guendouz, N. Lorrain, A. Moadhen, L. Haji, and M. Oueslati, "Spectroscopy studies of functionalized oxidized porous silicon surface for biosensing applications," Mater. Chem. Phys. 128, 151-156 (2011).

11. B. H. King, T. Wong, and M. J. Sailor, "Detection of pure chemical vapors in a thermally cycled porous silica photonic crystal," Langmuir 27, 8576-8585 (2011).

12. J. S. Ye and Z. Li, "A method for the measurement of methane gas based on multi-beam interferometry," J. Opt. Soc. Korea 17, 481-485 (2013).

13. L. Moretti, I. Rea, L. De Stefano, and I. Rendina, "Periodic versus a periodic: Enhancing the sensitivity of porous silicon based optical sensors," Appl. Phys. Lett. 90, 191112 (2007).

14. S. H. Park, Y. Y. Kim, and K. W. Lee, "Sensitivity improvement of free-standing porous silicon rugate filters for isopropanol vapor detection by applying lateral pressure differences," Sens. Actuators B 176, 437-442 (2013).

15. T. C. Do, H. Bui, T. V. Nguyen, T. A. Nguyen, T. H. Nguyen, and V. H. Pham, "A microcavity based on a porous silicon multilayer," Adv. Nat. Sci.: Nanosci. Nanotechnol. 2, 035001 (2011).

16. J. Chapron, S. A. Alekseev, V. Lysenko, V. N. Zaitsev, and D. Barbier, "Analysis of interaction between chemical agents and porous Si nanostructures using optical sensing properties of infra-red Rugate filters," Sens. Actuators B 120, 706-711 (2007).

17. M. S. Salem, M. J. Sailor, K. Fukami, T. Sakka, and Y. H. Ogata, "Sensitivity of porous silicon rugate for chemical vapor detection," J. Appl. Phys. 103, 083516 (2008).

18. N. A. Lange, Lange's Handbook of Chemistry, J. A. Dean, 15th ed. (Mc Graw-Hill, INC, ISBN 0-07-016384-7, 1999).

19. T. S. Pham, H. Bui, T. V. Nguyen, Q. M. Ngo, T. A. Nguyen, T. H. C. Hoang, T. C. Do, Q. H. Le, and V. H. Pham, "Liquid sensors based on porous silicon microcavity," Proc. Adv. Opt. Photo. Spec. \& Appl. VII, ISSN 18594271, 754-759 (2013). 